\title{
Chemical Constituents and Their Bioactivities of Mushroom Phellinus rhabarbarinus
}

Tao Feng, ${ }^{\dagger}$ Jin-Long Cai, ${ }^{\dagger}$ Xue-Mei Li, ${ }^{\dagger}$ Zhong-Yu Zhou, ${ }^{\S}$ Zheng-Hui Li, ${ }^{*, \dagger}$ and Ji-Kai Liu* ${ }^{* \dagger}$

${ }^{\dagger}$ College of Pharmacy, South-Central University for Nationalities, Wuhan 430074, China, ${ }^{\star}$ School of Agriculture and Biological Technic, Yunnan Agricultural University, Kunming 650201, China, ${ }^{\S}$ Key Laboratory of Plant Resources Conservation and Sustainable Utilization, South China Botanical Garden, Chinese Academy of Sciences Guangzhou 510650, China.

\section{Contents}

Page S2-S9. NMR and HR-ESI-MS spectra of phellibarin A (1).

Page S10-S17. NMR and HR-ESI-MS spectra of phellibarin B (2).

Page S18-S25. NMR and HR-ESI-MS spectra of phellibarin C (3). 
${ }^{1} \mathrm{H}$ NMR spectrum of phellibarin A (1)

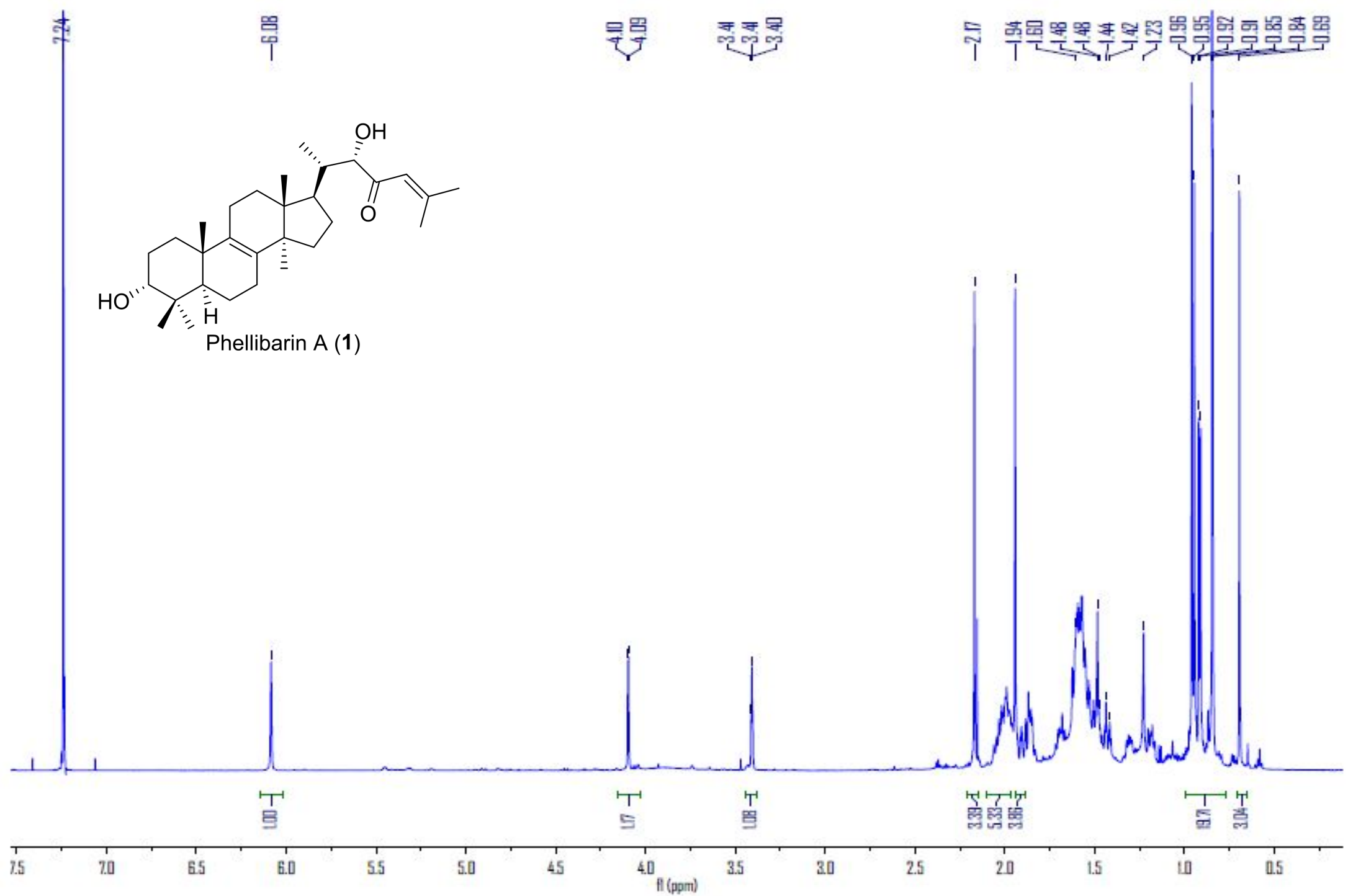




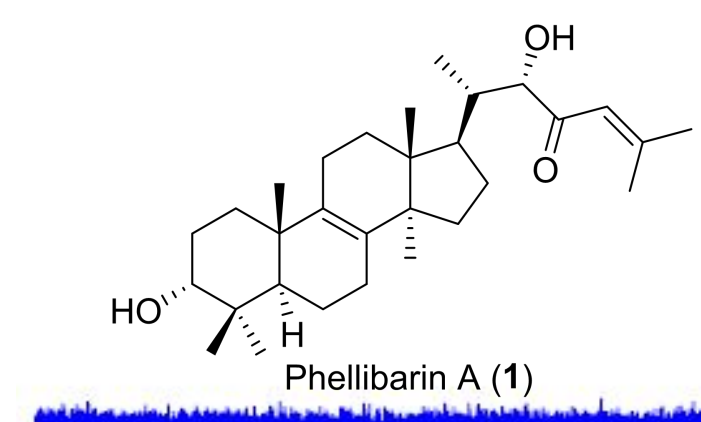

${ }^{13} \mathrm{C}$ NMR spectrum of phellibarin A (1)
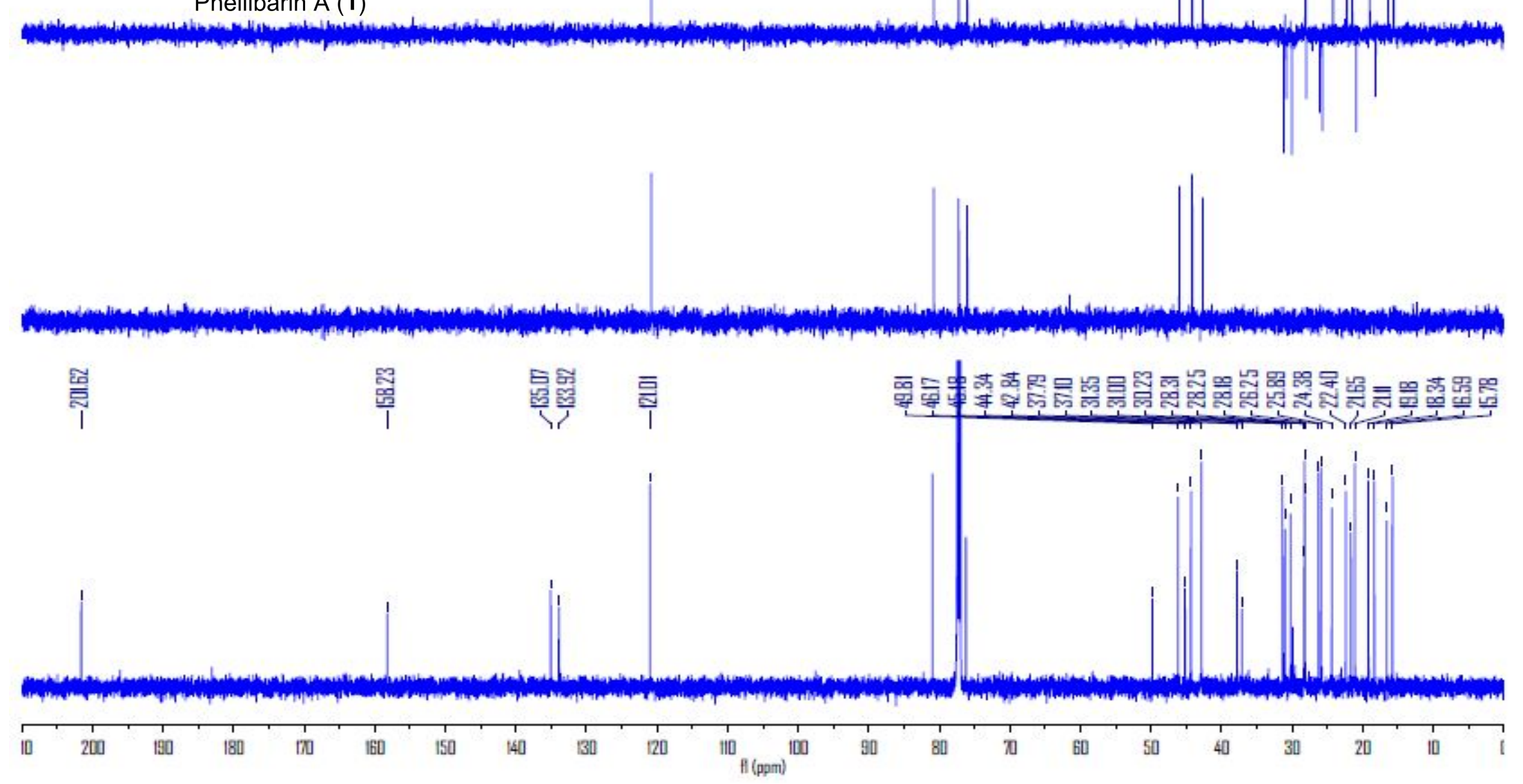


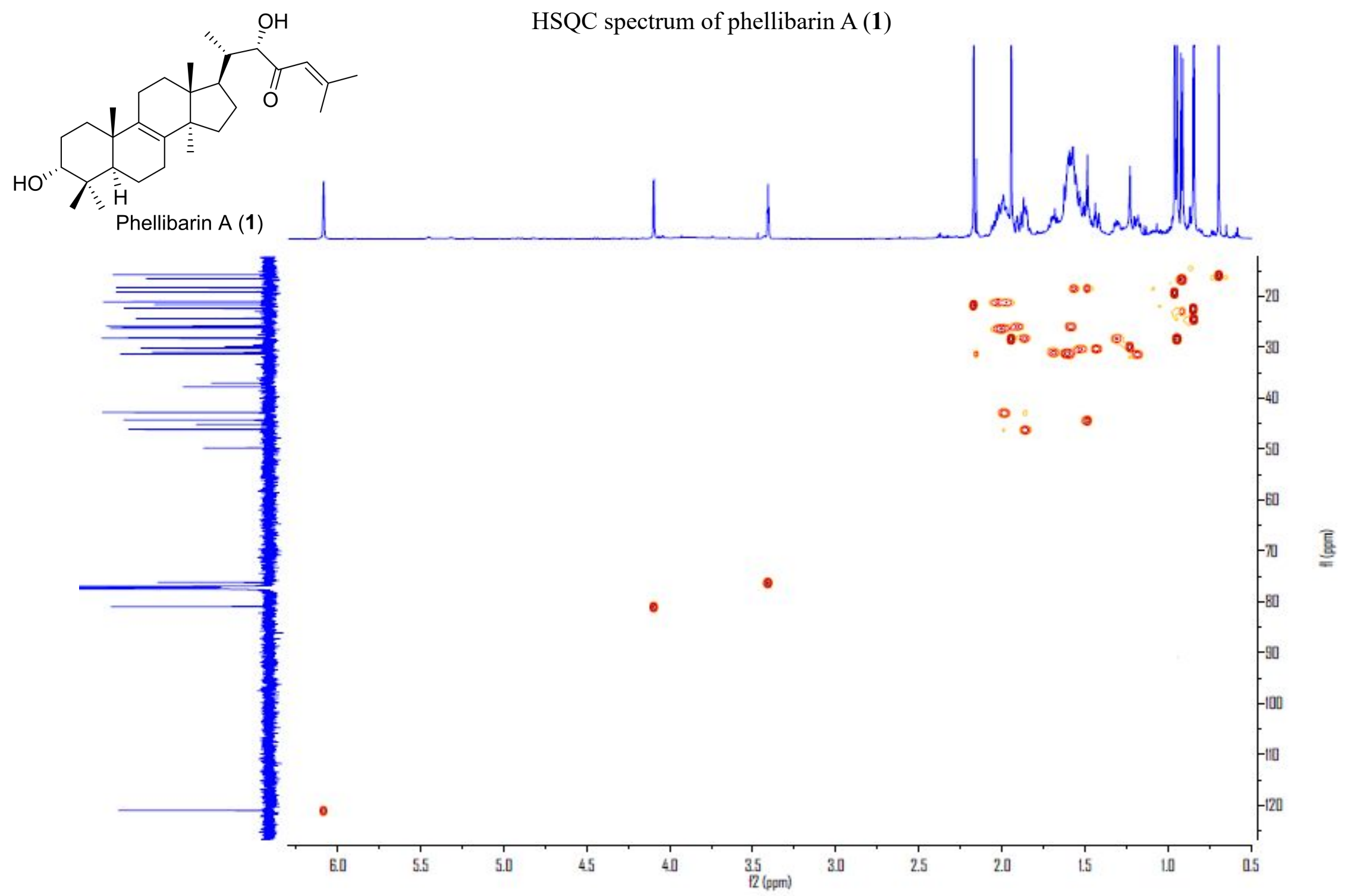




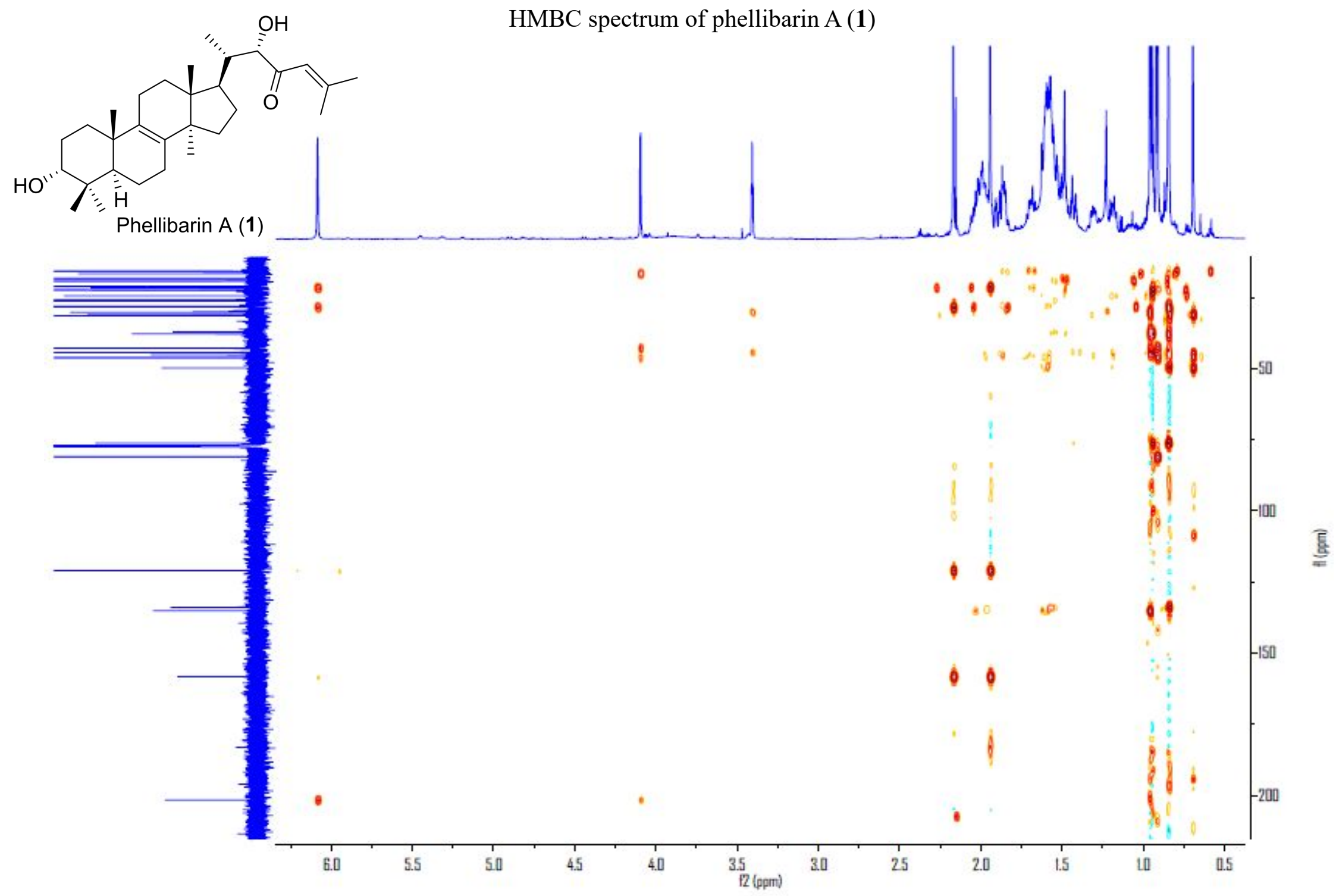




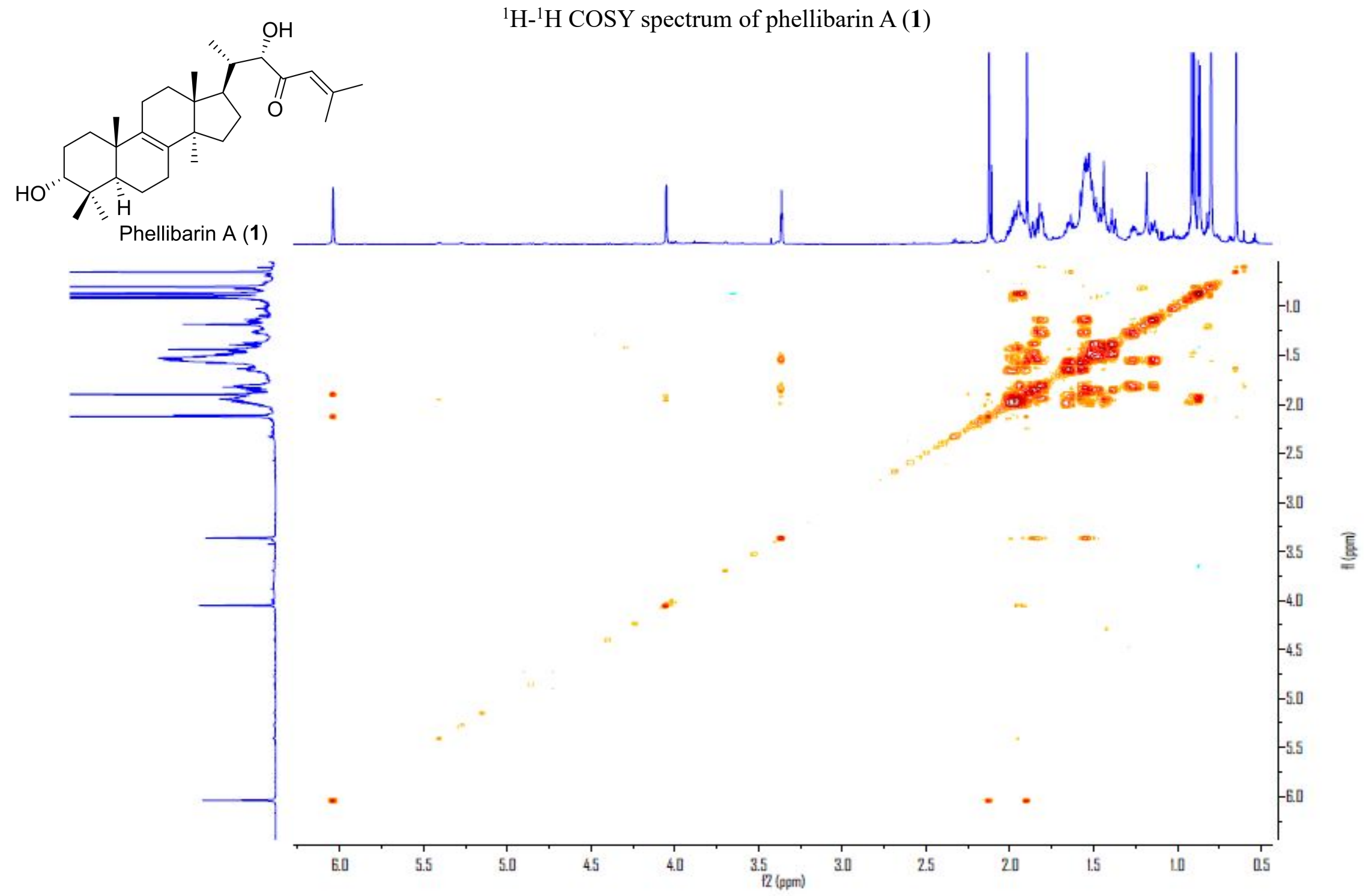




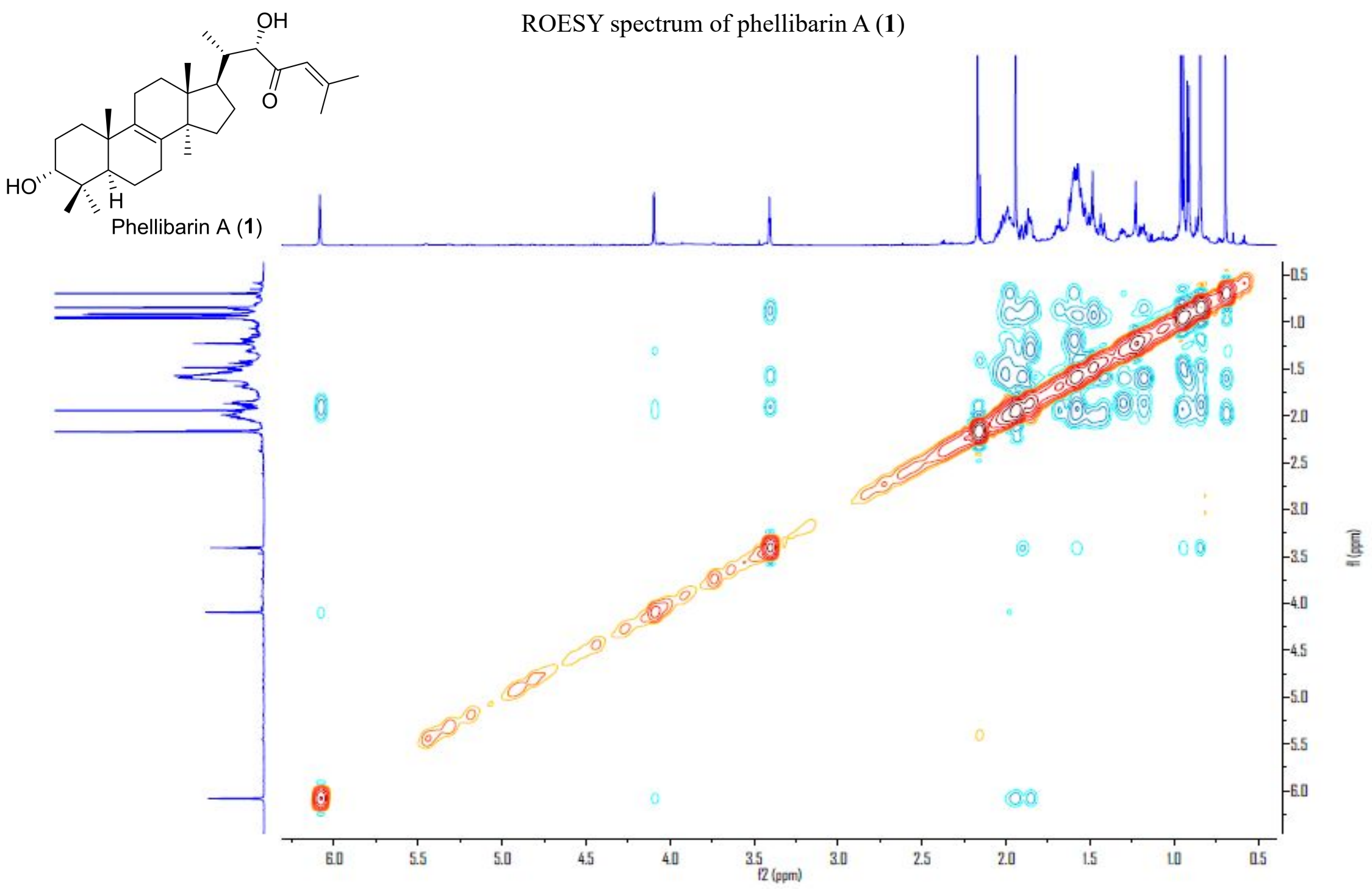


Positive ESIMS of phellibarin A (1)

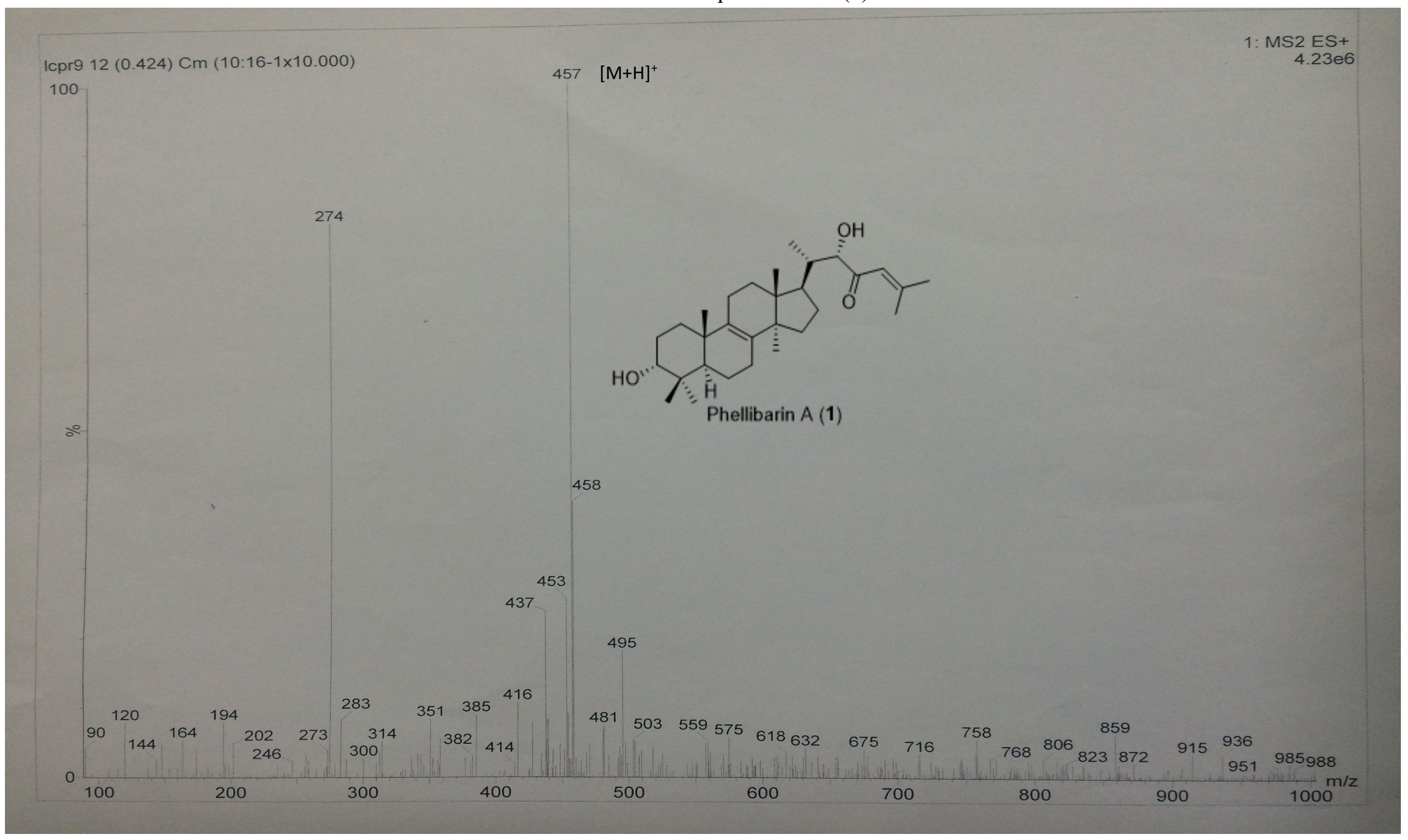


HR-ESI-MS of phellibarin A (1)

Fragmentor Voltage Collision Energy Ionization Mode

$$
135
$$

0

$$
\text { ESI }
$$

$\times 103+$ Scan (0.186-0.219 min, 3 Scans) Icpr9+2.d Subtract (2)


Peak List

\begin{tabular}{|l|r|l|}
\hline$m / z$ & $\mathbf{z}$ & Abund \\
\hline 218.2117 & & 8950.67 \\
\hline 274.2745 & 1 & 41302.79 \\
\hline 302.3057 & 1 & 9559.1 \\
\hline 318.3008 & 1 & 20927.25 \\
\hline 432.2378 & 1 & 9879.38 \\
\hline 437.1936 & 1 & 16110.32 \\
\hline 453.1674 & 1 & 27130.4 \\
\hline 471.3464 & 1 & 9123.1 \\
\hline Formula Calculator Element Limits \\
\begin{tabular}{|l|r|c|}
\hline Element & Min & Max \\
\hline C & \multicolumn{3}{|r|}{3} & 60 \\
\hline H & \multicolumn{3}{|r|}{0} & 120 \\
\hline O & 0 & 30 \\
\hline
\end{tabular}
\end{tabular}

Formula Calculator Results

Formula

CalculatedMz CalculatedMass 456.3604 479.3496 \begin{tabular}{|l|l} 
Mz & Diff. (mDa)
\end{tabular} Diff. (ppm) DBE

$\mathrm{HO}$

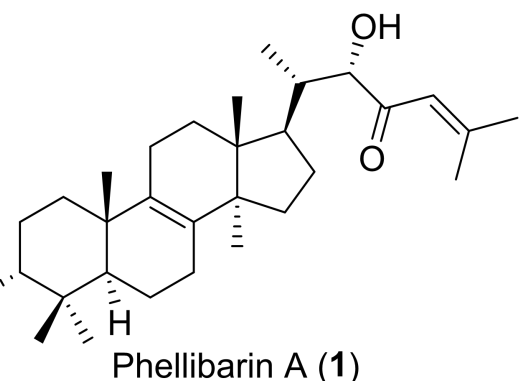


${ }^{1} \mathrm{H}$ NMR spectrum of phellibarin B (2)

罩

学㽞

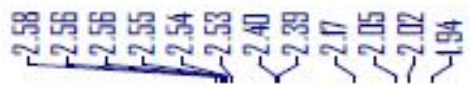

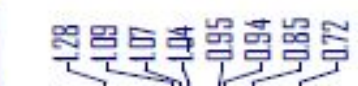

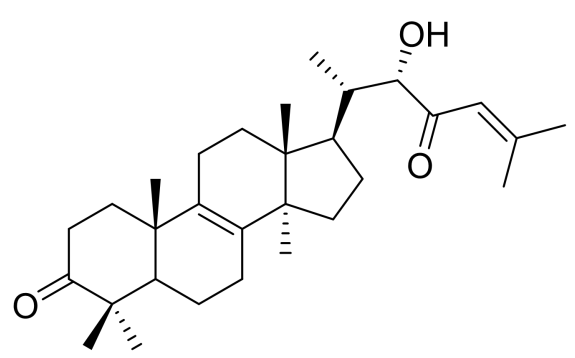

Phellibarin B (2)

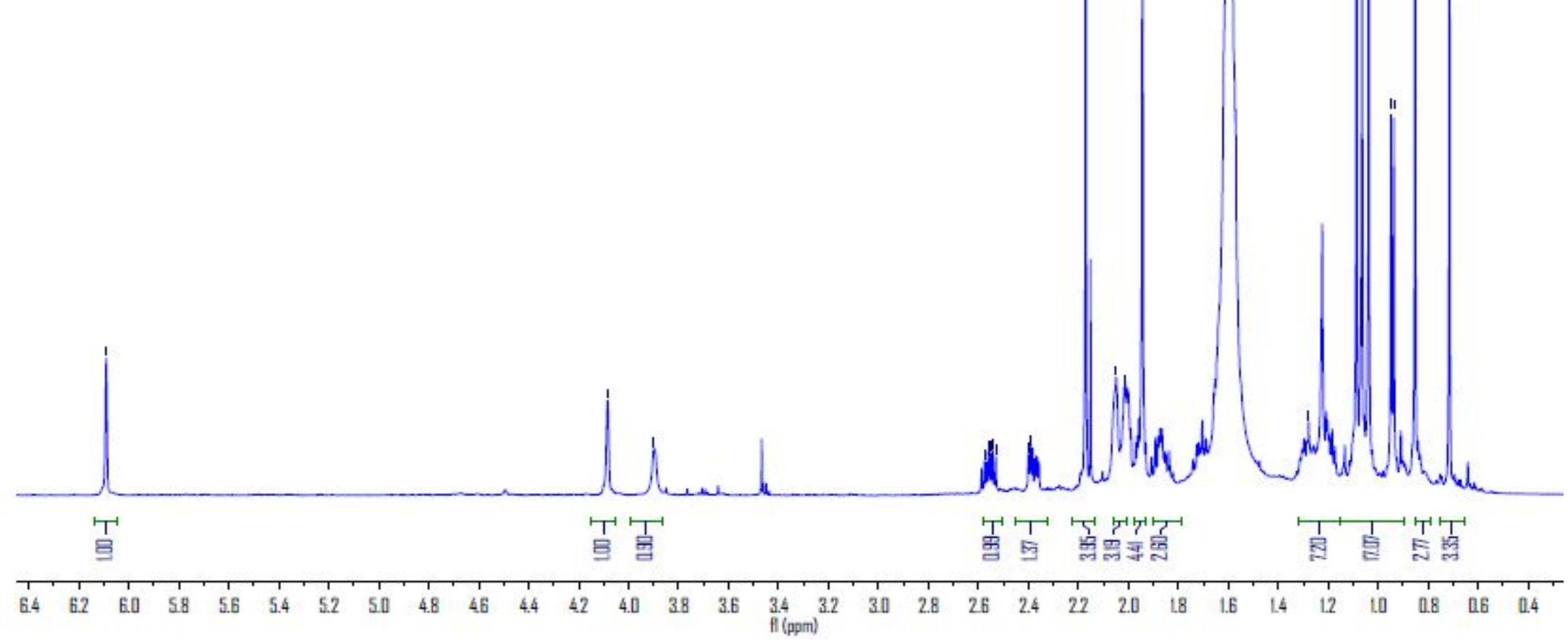




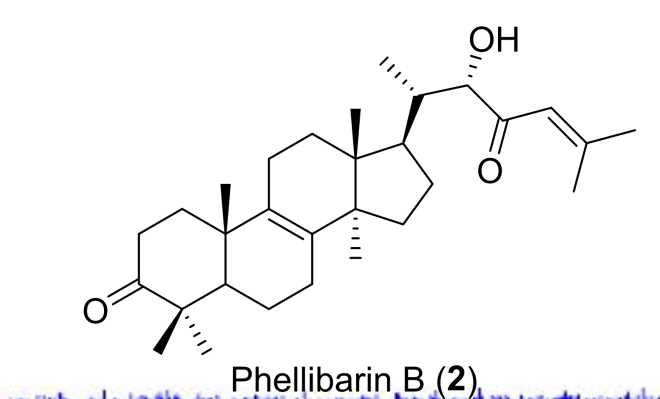

${ }^{13} \mathrm{C}$ NMR spectrum of phellibarin B (2)

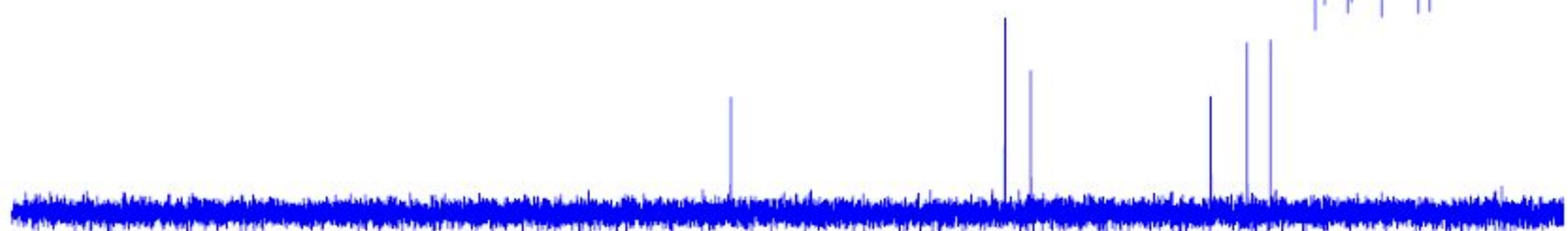

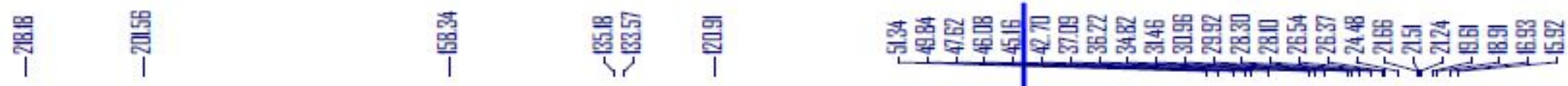






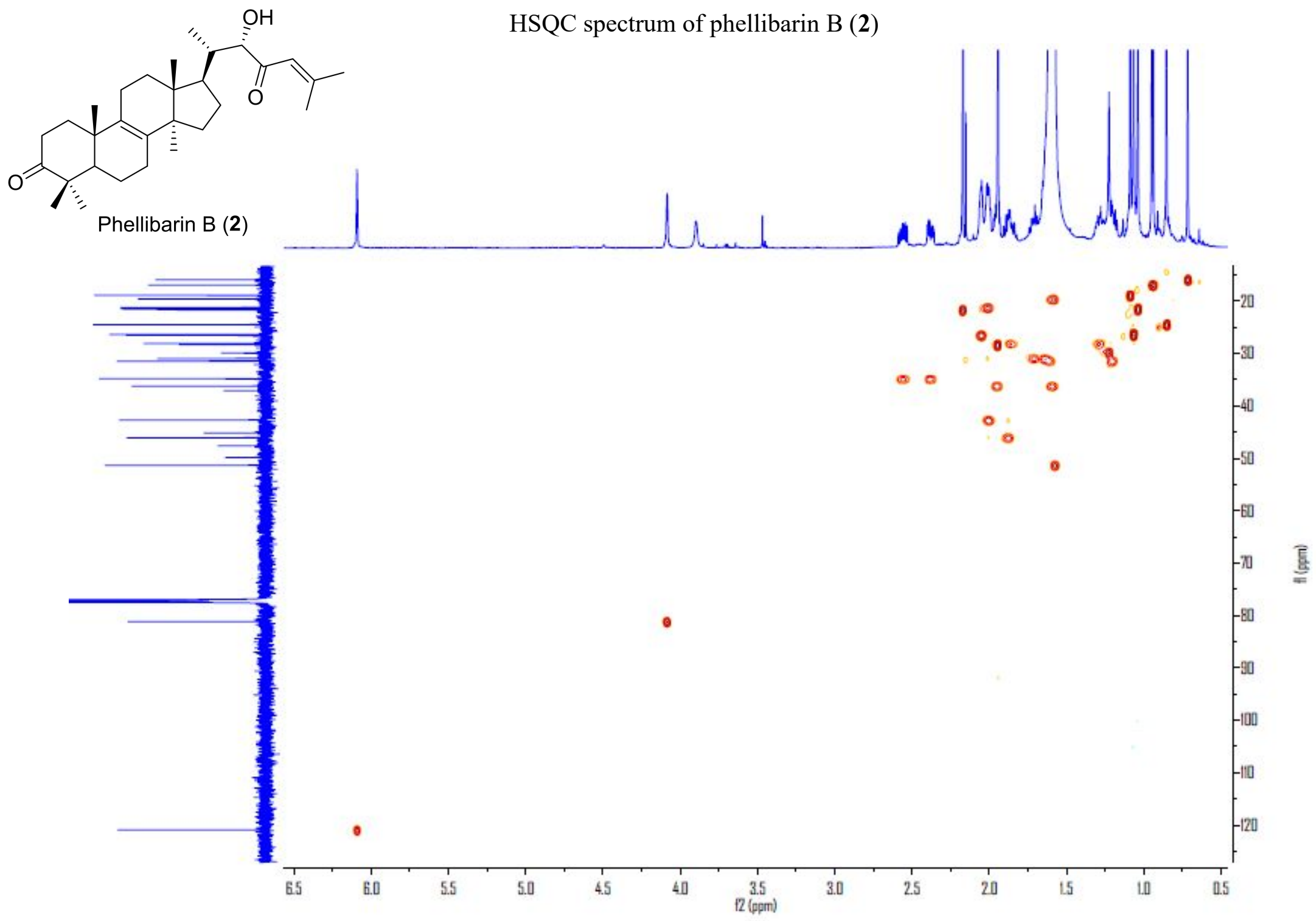




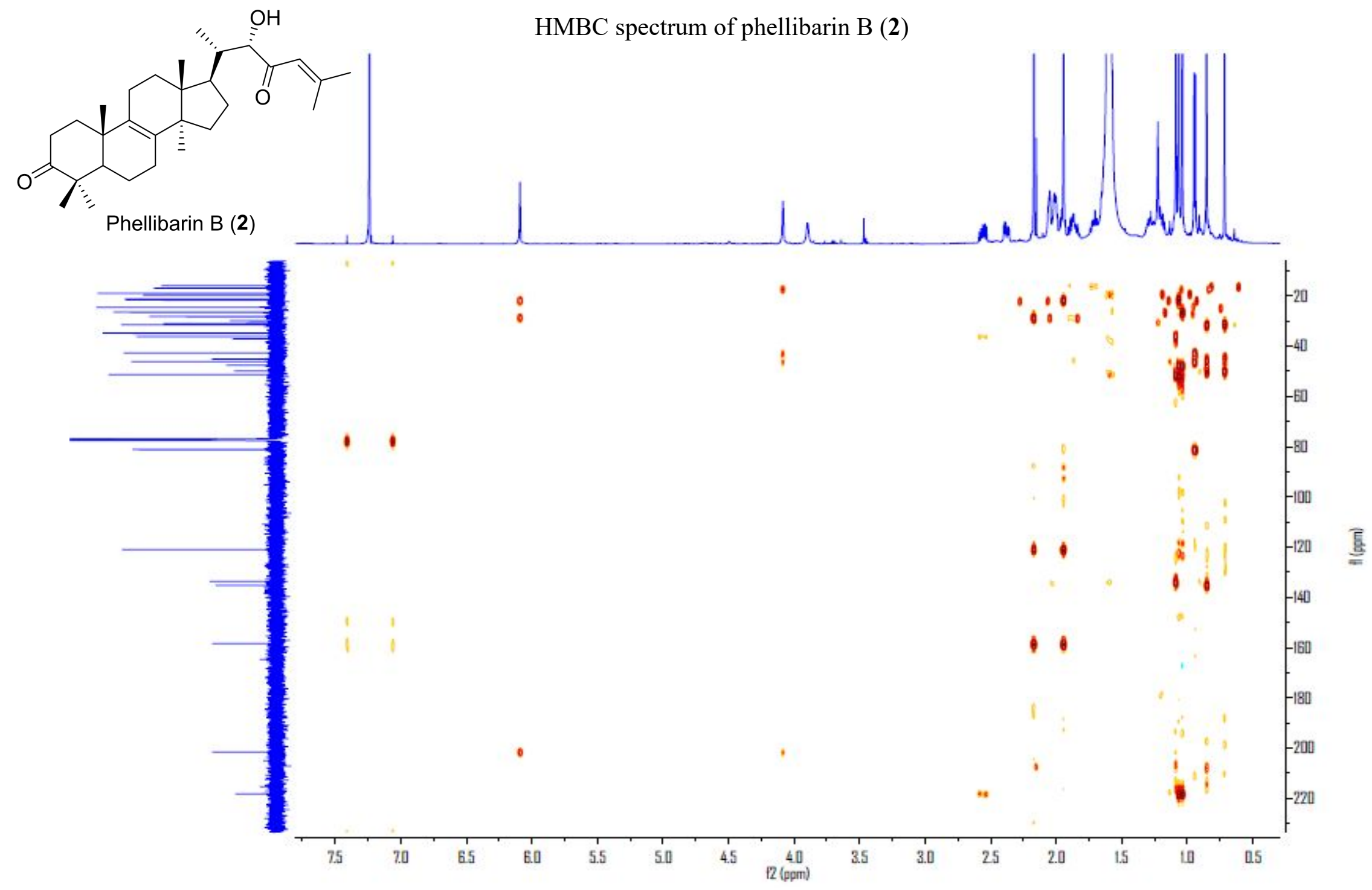




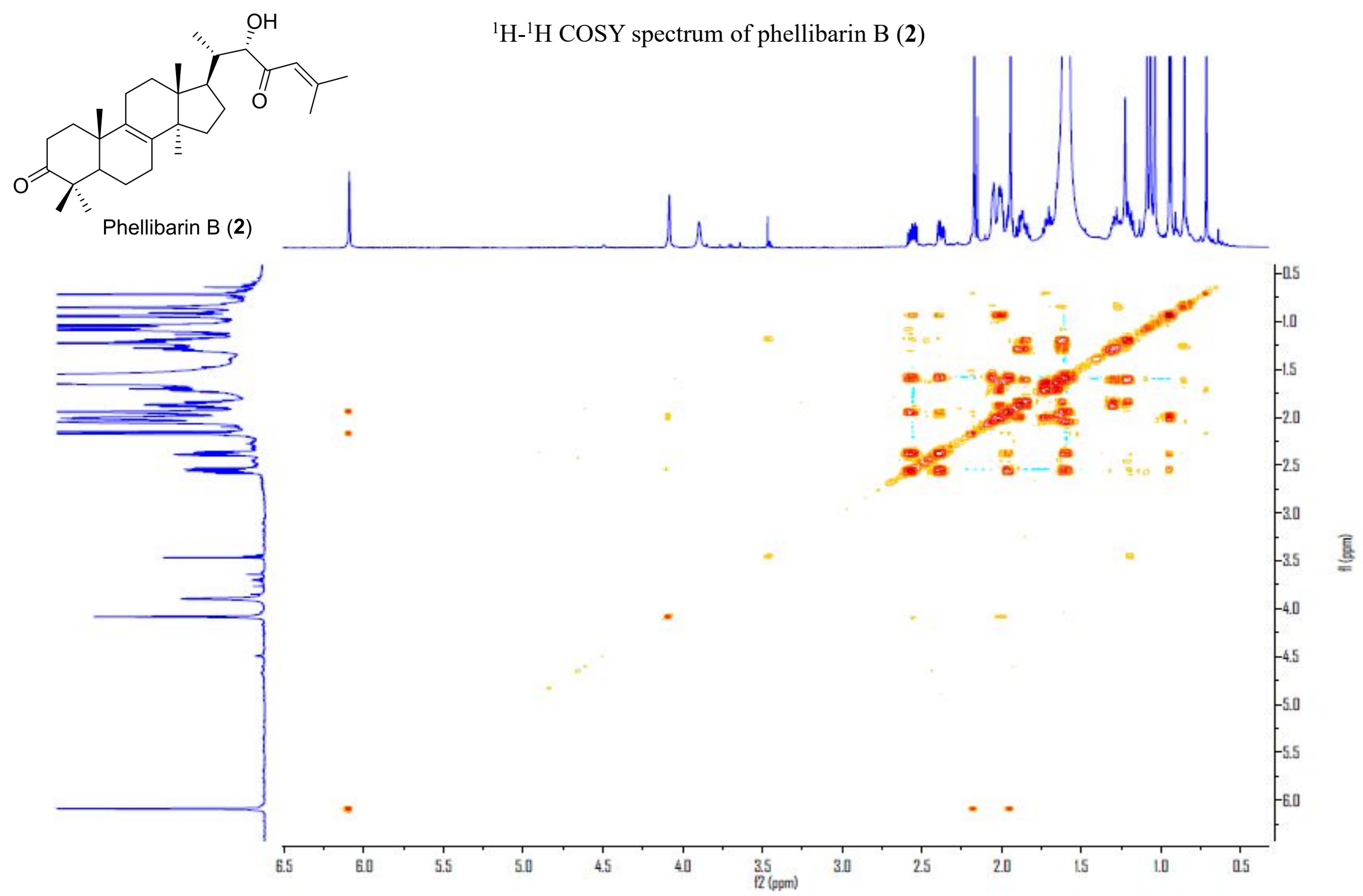




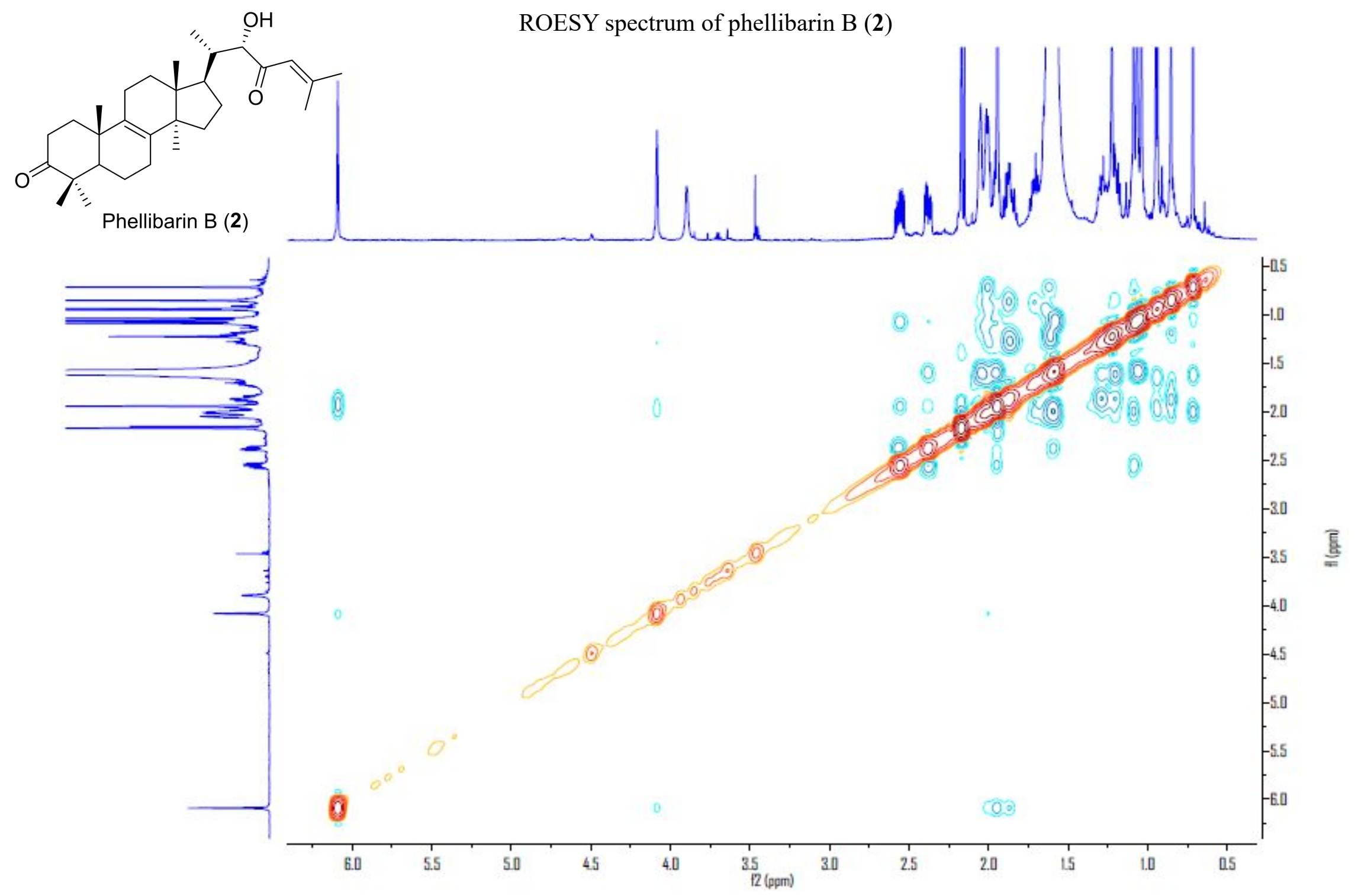

S15 
Positive ESIMS of phellibarin B (2)

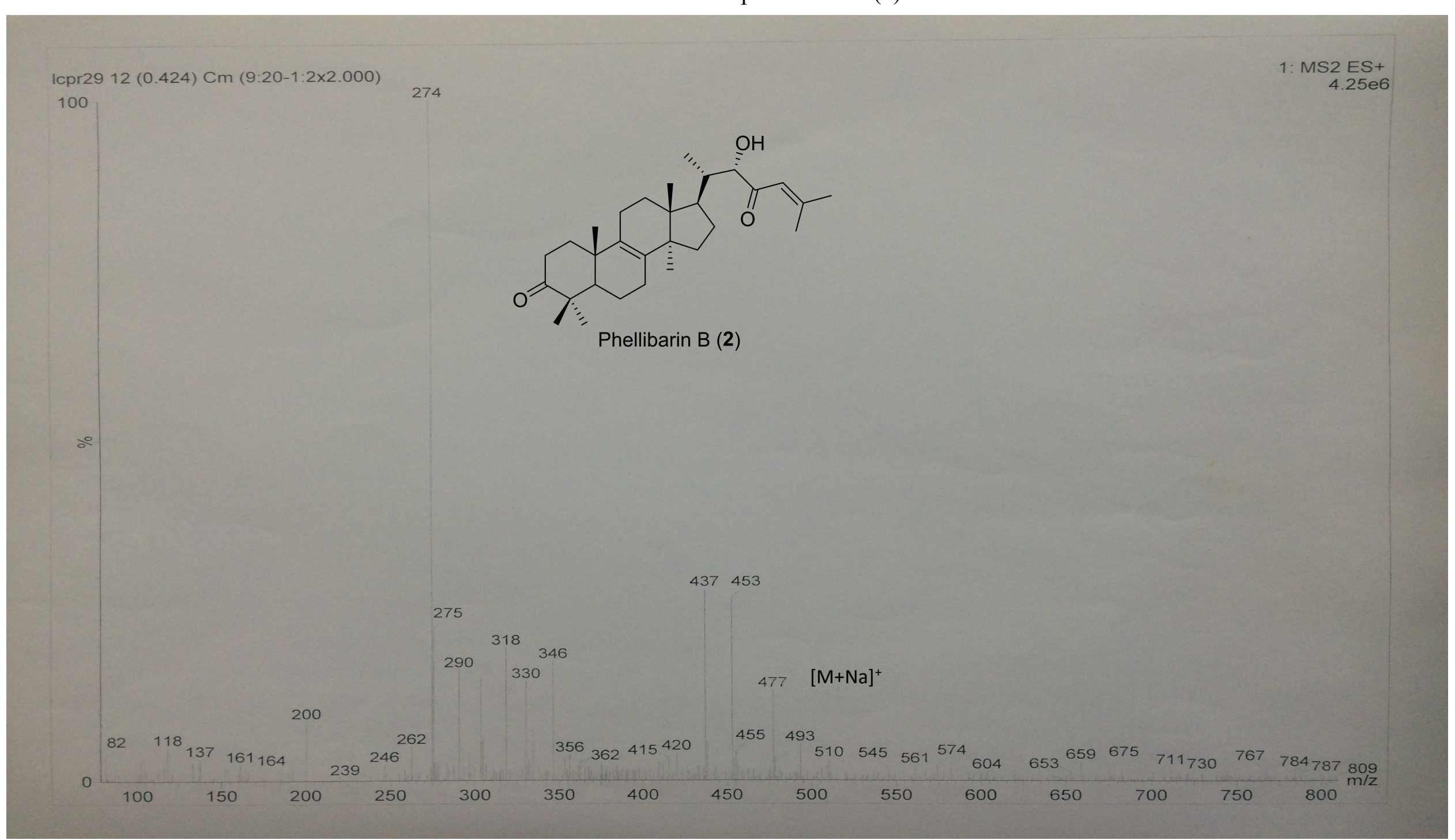


HR-ESI-MS of phellibarin B (2)

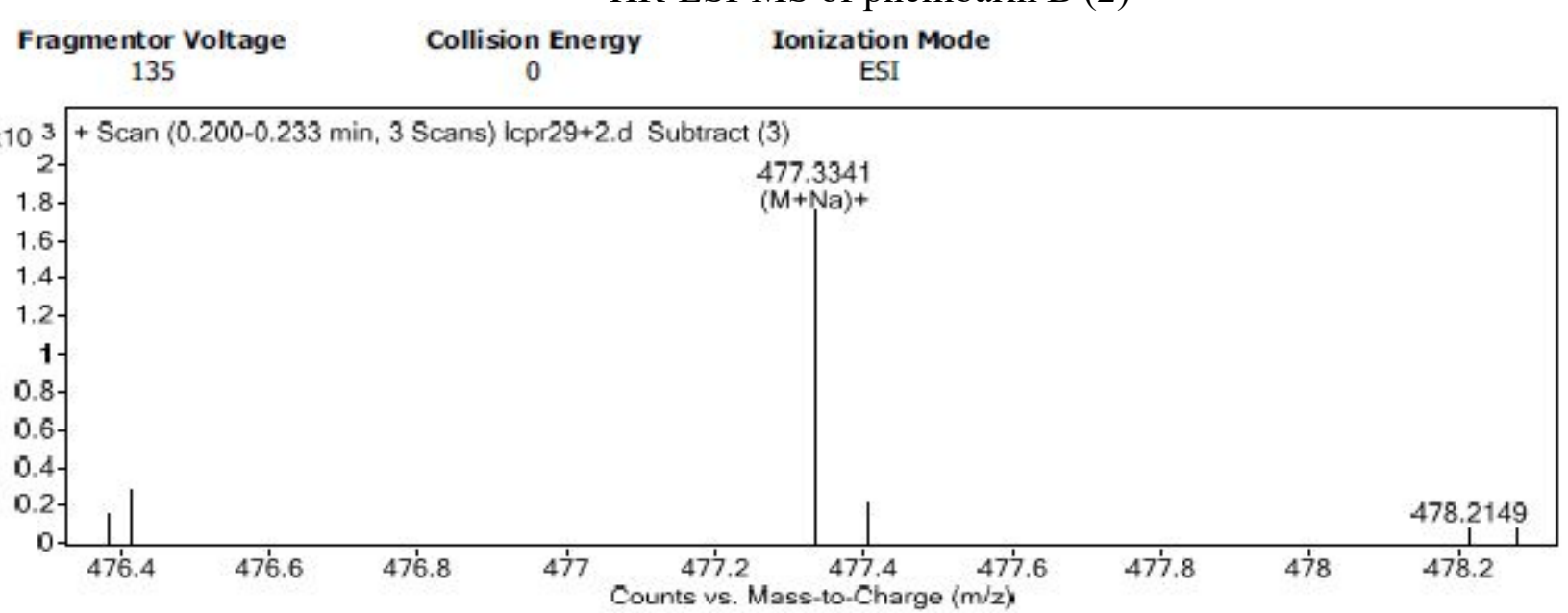

\begin{tabular}{|c|c|c|c|}
\hline \multirow{2}{*}{\multicolumn{4}{|c|}{ Peak List }} \\
\hline & \multicolumn{3}{|c|}{\begin{tabular}{l|l|}
$z$ & Abund \\
\end{tabular}} \\
\hline 246.243 & 1 & \multicolumn{2}{|c|}{74479.31} \\
\hline 274.2746 & 1 & \multicolumn{2}{|c|}{882326.31} \\
\hline 275.2777 & 1 & \multicolumn{2}{|c|}{141633.73} \\
\hline 302.3059 & 1 & \multicolumn{2}{|c|}{285177.97} \\
\hline \begin{tabular}{|l|}
318.3009 \\
\end{tabular} & 1 & \multicolumn{2}{|c|}{380882.94} \\
\hline 330.337 & 1 & \multicolumn{2}{|c|}{131993.31} \\
\hline 346.3319 & 1 & \multicolumn{2}{|c|}{129048.77} \\
\hline 362.3267 & & \multicolumn{2}{|c|}{87688.57} \\
\hline \multirow{2}{*}{\multicolumn{4}{|c|}{$\begin{array}{l}\text { Formula Calculator Element } \\
\begin{array}{|l|l|l|}\text { Element } & \text { Min } & \text { Max } \\
\end{array}\end{array}$}} \\
\hline & & & \\
\hline C & & 3 & 60 \\
\hline $\mathrm{H}$ & & 0 & 120 \\
\hline 0 & & 0 & 30 \\
\hline
\end{tabular}

Formula Calculator Results

\begin{tabular}{|l|c|c|c|r|r|r|}
\hline Formula & CalculatedMass & CalculatedMz & Mz & Diff. (mDa) & Diff. (ppm) & DBE \\
\hline $\mathrm{C} 30 \mathrm{H} 46 \mathrm{O} 03$ & 454.3447 & 477.3339 & 477.3341 & 0.6 & 1.3 & 8.0000 \\
\hline
\end{tabular}


${ }^{1} \mathrm{H}$ NMR spectrum of phellibarin C (3)

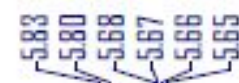

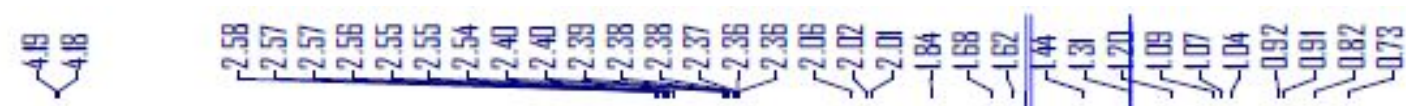

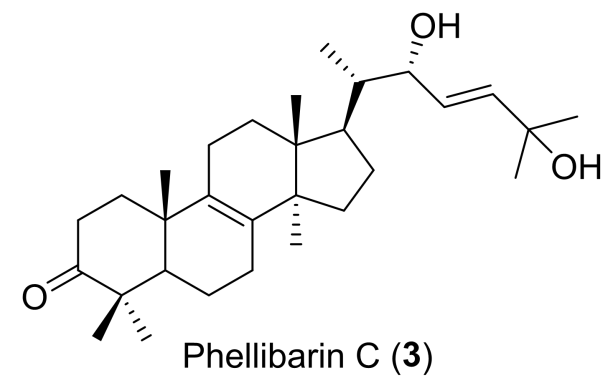

Phellibarin C (3)
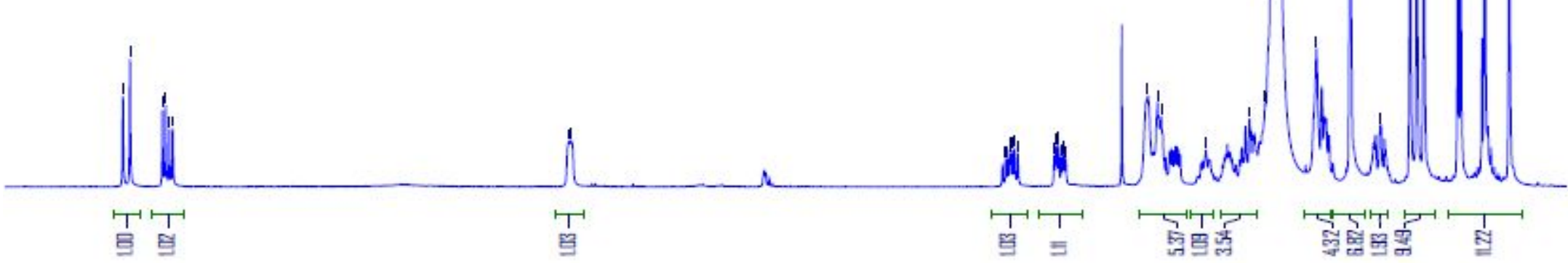

$\begin{array}{llllllllllllllllllllllllllllllllllllllll}6.2 & 6.0 & 5.8 & 5.6 & 5.4 & 5.2 & 5.0 & 4.8 & 4.6 & 4.4 & 4.2 & 4.0 & 3.8 & 3.6 & 3.4 & 3.2 & 3.0 & 2.8 & 2.6 & 2.4 & 2.2 & 2.0 & 1.8 & 1.6 & 1.4 & 1.2 & 1.0 & 0.8 & 0.6\end{array}$ 

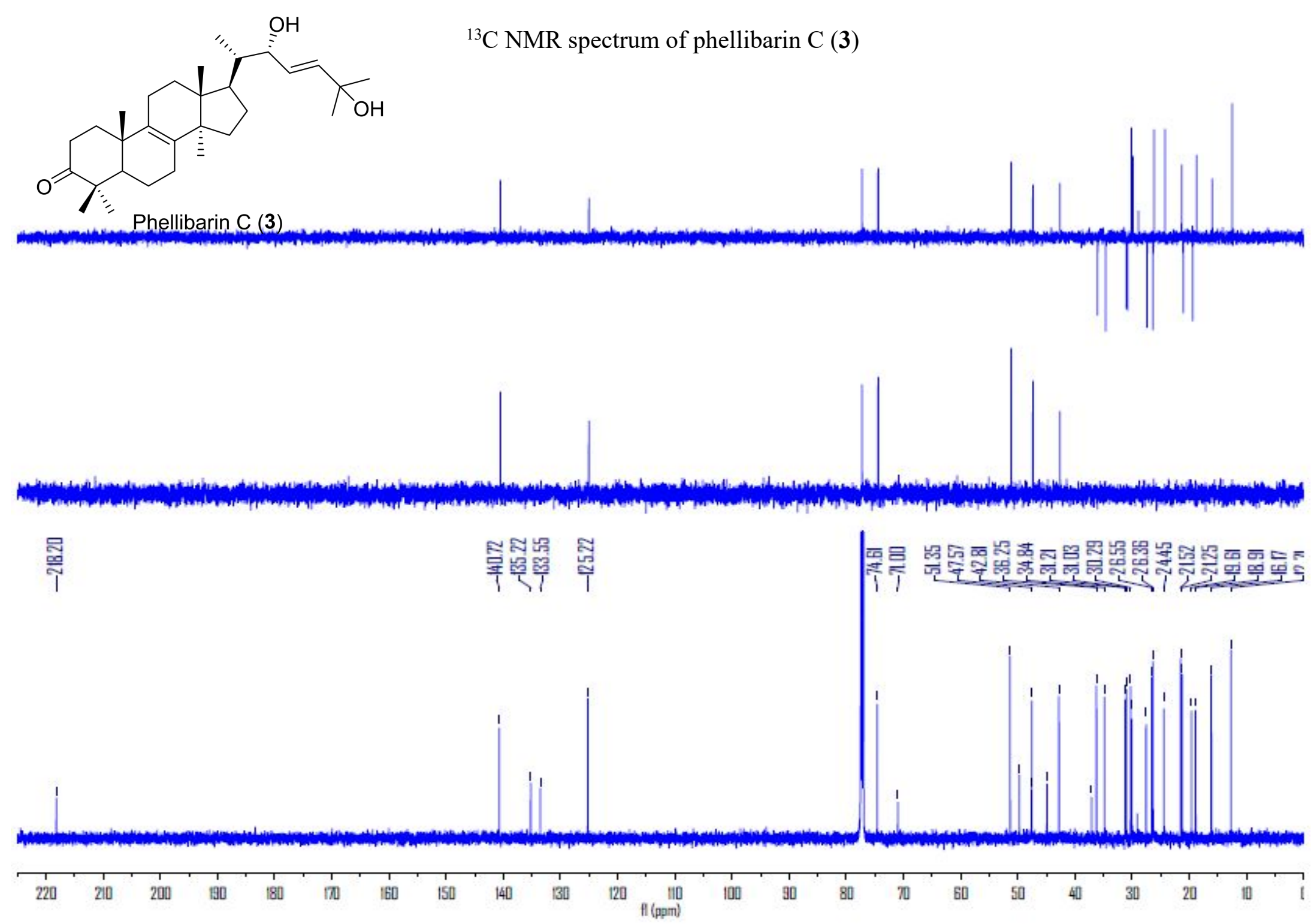


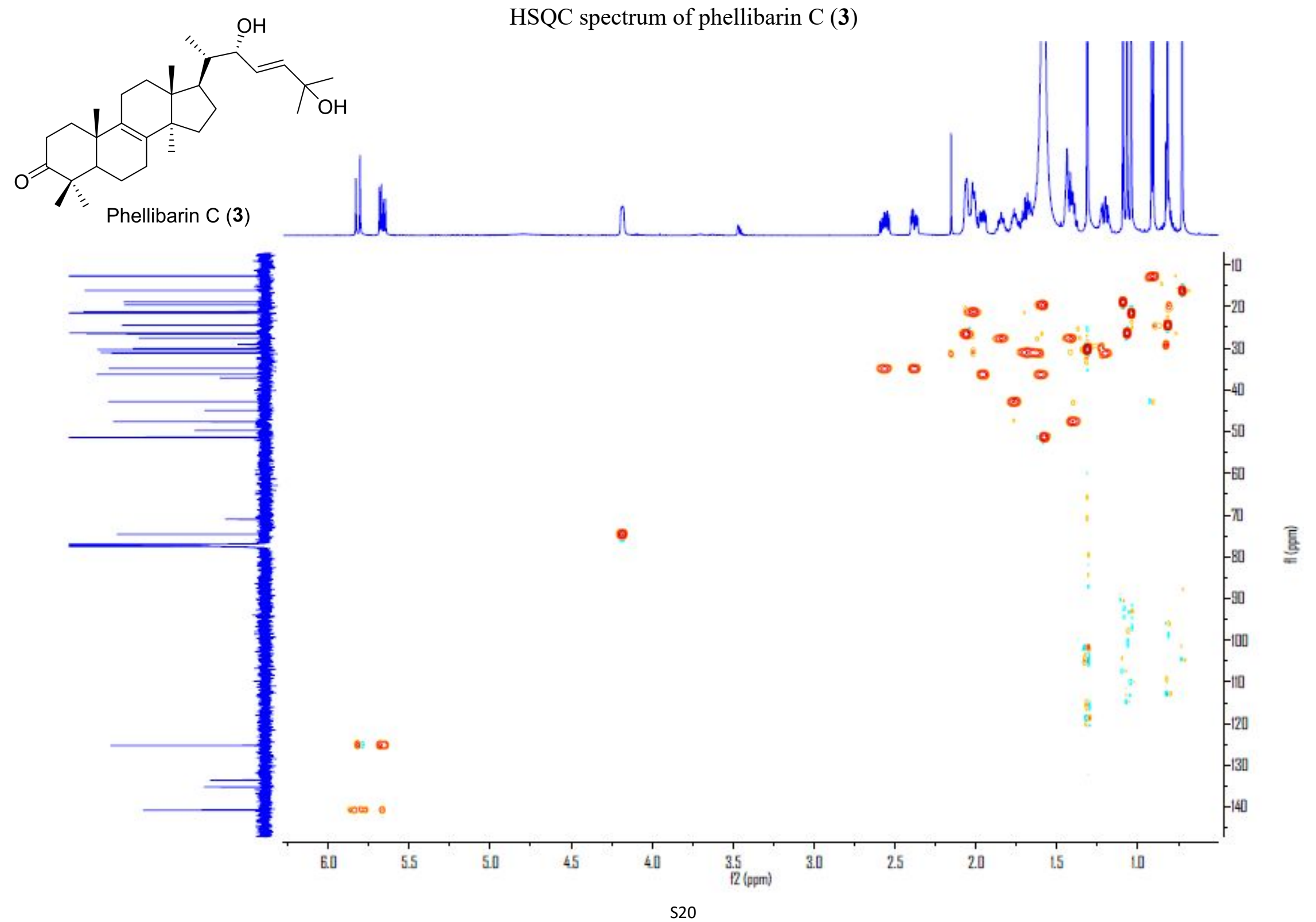




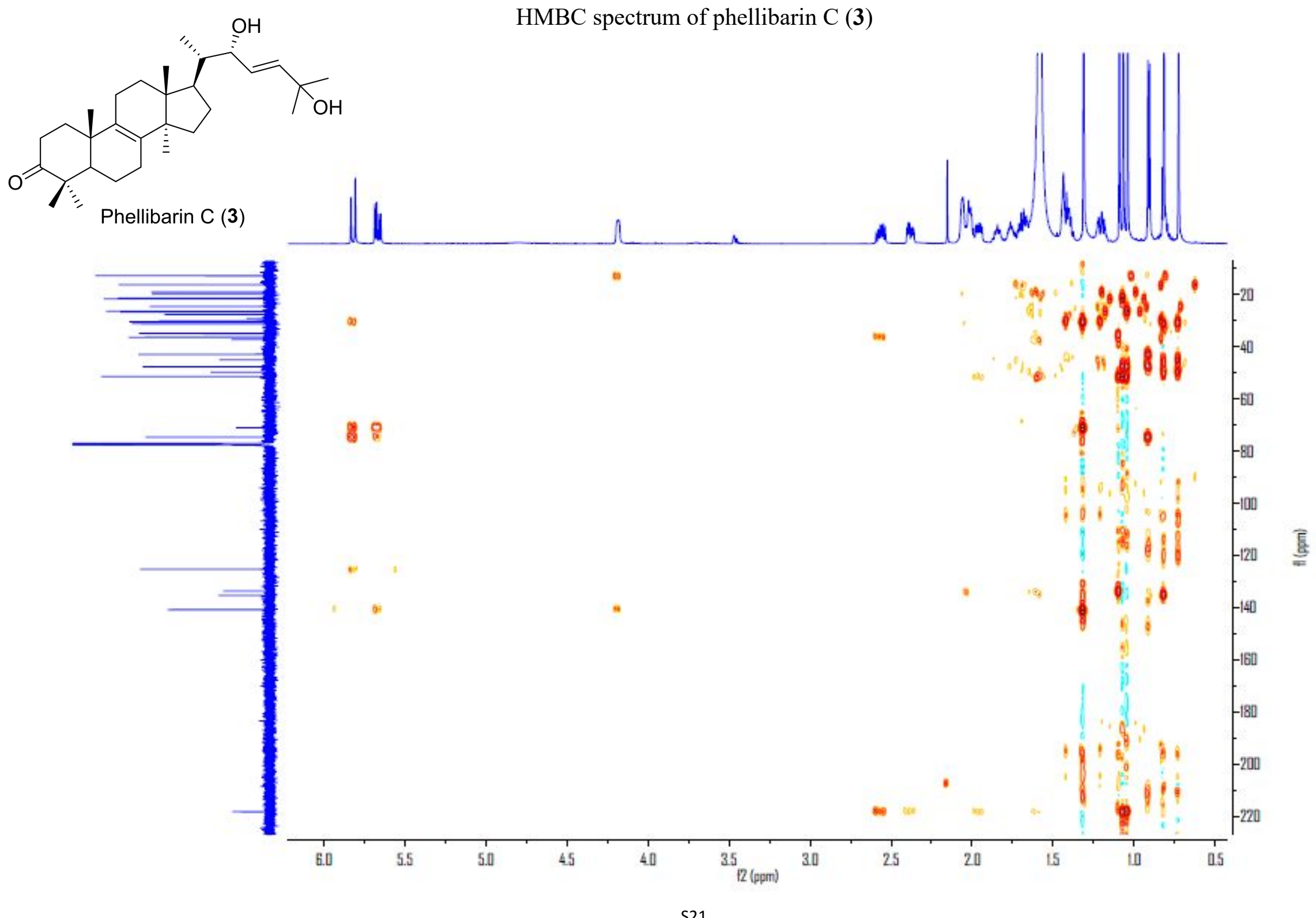




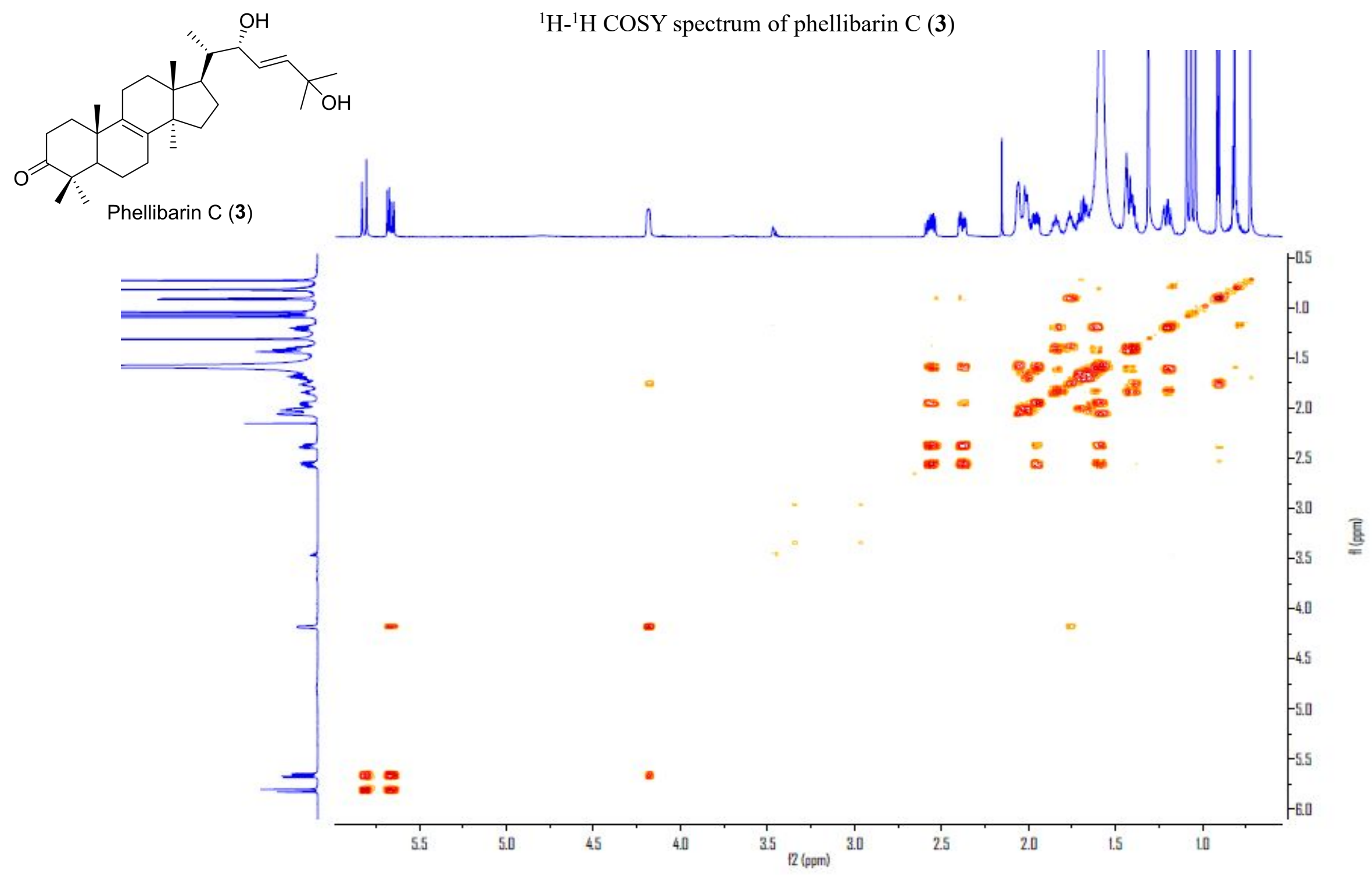




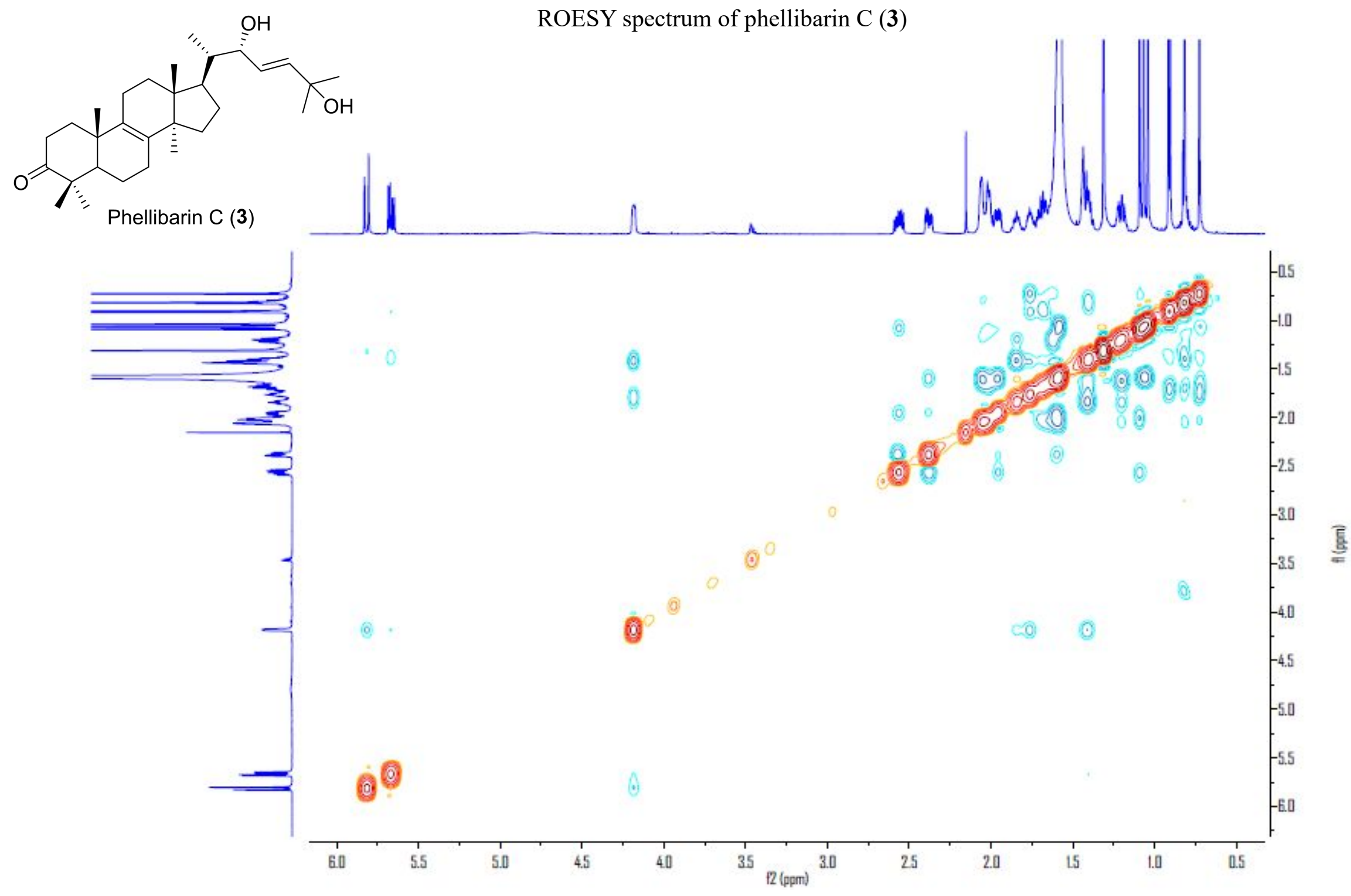


Positive ESIMS of phellibarin C (3)

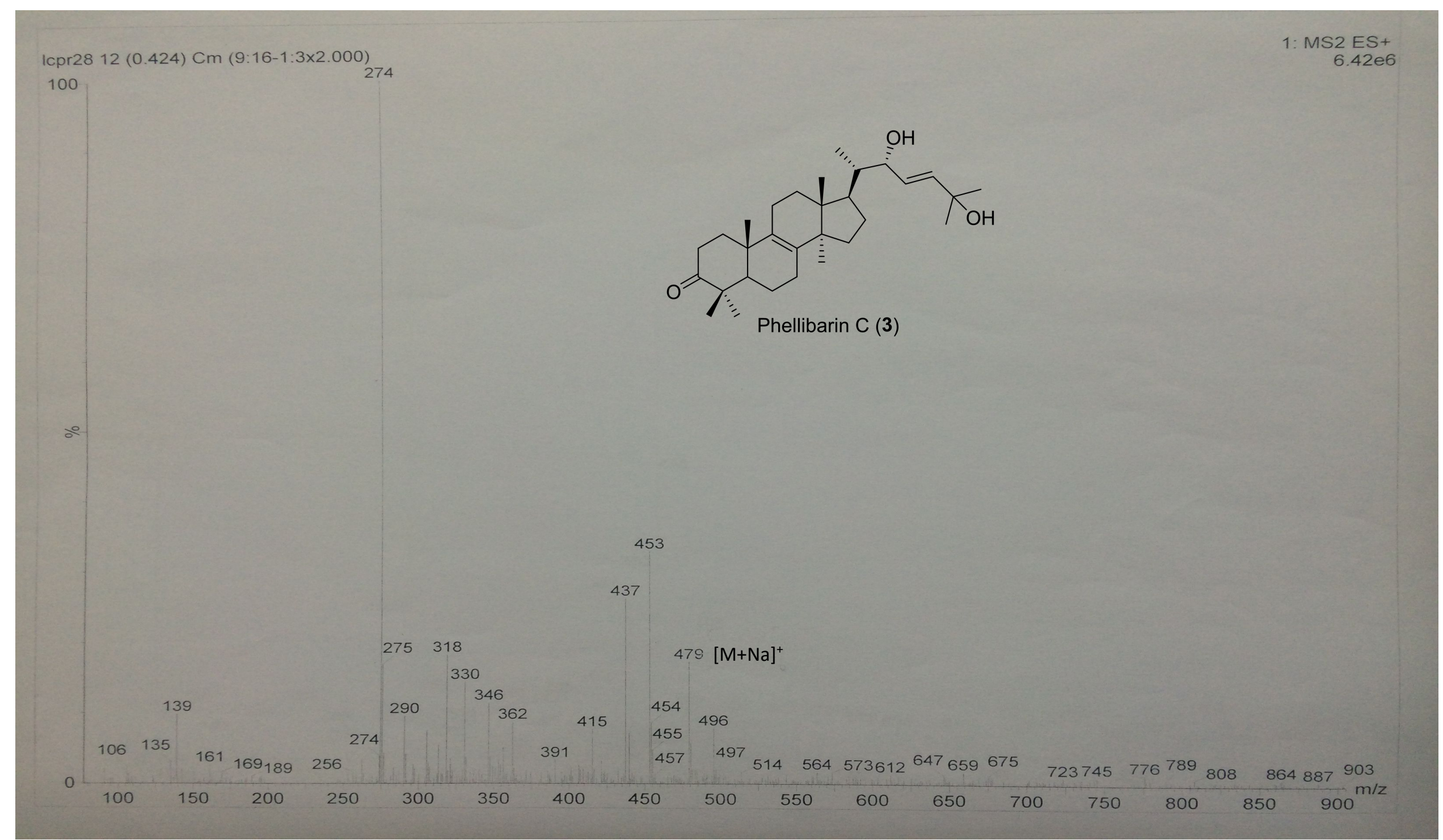


HR-ESI-MS of phellibarin C (3)

$\begin{array}{ccc}\text { Fragmentor Voltage } & \text { Collision Energy } & \text { Ionization Mode } \\ 135 & \text { ESI }\end{array}$

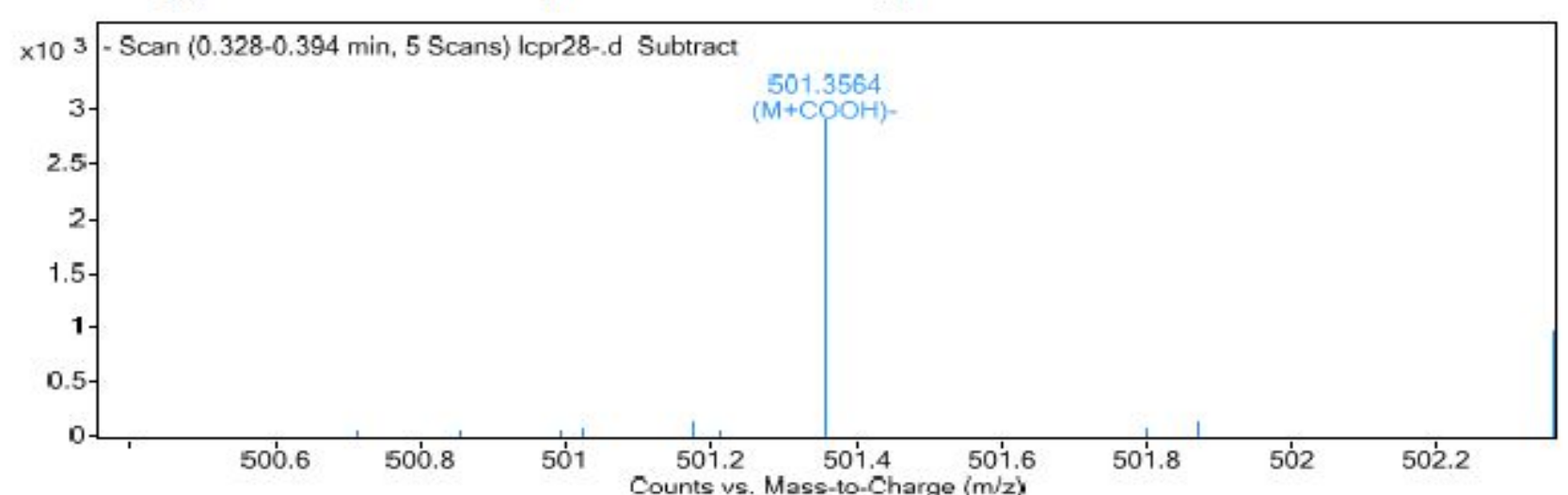

Peak List

\begin{tabular}{|l|l|l|l|l|}
\hline $\boldsymbol{m} / \boldsymbol{z}$ & $\mathbf{z}$ & Abund & Formula & Ion \\
\hline 89.0242 & & 3088.72 & & \\
\hline 112.9854 & 1 & 11438.01 & & \\
\hline 255.2321 & 1 & 7317.34 & & \\
\hline 256.2355 & 1 & 1763.83 & & \\
\hline 283.2632 & 1 & 6240.49 & & \\
\hline 499.1699 & 1 & 2868.72 & & \\
\hline 501.3564 & 1 & 2898.46 & C30 H48 O3 & $(\mathrm{M}+\mathrm{COOH})-$ \\
\hline 1033.9845 & 1 & 7131.61 & & \\
\hline
\end{tabular}

\begin{tabular}{l|c|c|c|}
\hline 1033.9845 & 1 & 7131.61 & \\
\hline Formula Calculator Element Limits
\end{tabular}

Element 1 Min

\begin{tabular}{|l|r|c|}
\hline $\mathrm{C}$ & 3 & 60 \\
\hline $\mathrm{H}$ & 0 & 120 \\
\hline $\mathrm{O}$ & 0 & 30 \\
\hline
\end{tabular}

Formula Calculator Results

\begin{tabular}{l|l} 
Formula & CalculatedMass
\end{tabular}

$\mathrm{C} 30 \mathrm{H} 48 \mathrm{O3}$

CalculatedMass $\quad$ CalculatedMz

456.3604

501.3585

\begin{tabular}{l|l} 
Mz & Diff. (mDa)
\end{tabular}

4

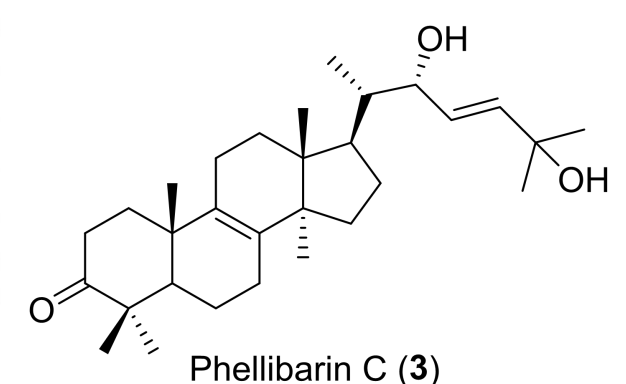

Phellibarin C (3) 